\title{
XVII.
}

\section{Beitrag zur chronischen, ankylosirenden Entzündung der Wirbelsäule und der Hüftgelenke.}

\author{
Von \\ Prof. Dr. Valentini in Danzig, \\ frïher Privatdocent in Königşerg i./P.
}

(Nit 2 Abbildungen.)

Im Juni des Jahres $1896 \mathrm{kam}$ ein Patient in meine Beobachtung, der ein merkwärdiges, mir völlig unbekanntes Krankheitsbild bot. Die wesentlichsten Symptome waren eine absolute Unbeweglichkeit der Wirbelsäule und beider Hüftgelenke. Zur genaneren Feststellung des Befundes hatte Herr Professor Lichtheim die Güte, den Kranken in die medicinische Klinik aufzunehmen. -

Es wurde angenommen, dass der vorliegende Fall sich nicht mit einer bekannten Krankheit der Gelenke oder Knochen identificiren liesse und somit eine unbekannte Krankheit vorliege. Von einer Publication wurde Abstand genommen, da die Mittheilung eines vereinzelten Falles ohne autoptischen Befund wohl kaum einen Werth hat.

Im April 1898 ersehien dann eine Arbeit von Pierre Marie in der Revue de Médecine ${ }^{1}$ ): „Sur la hpondylose rhizomélique", über die Herr Professor Lichtheim in einer Versammlung der Professoren und Assistenten der chirurgischen und medicinischen Klinik Mittheilung machte mit dem Hinweis, dass der in Jahre 1896 beobachtete, nicht klassifizirbare Fall mit den von Pierre Marie unter obigem Titel mitgetheilten Fällen identisch sei.

Der Autor theilt in dieser Arbeit drei Fälle mit, die in den letzten 10 Jahren zu seiner Kenntniss gekommen sind.

Aus der genauen und eingehenden Schilderung sieht man, dass dieselben fast die ganz gleichen Symptome boten.

Im Alter von ungefähr zwanzig Jahl'ei beginnt die Krankheit mit Schmerzen in der Kreuzbein- und Hüftgegend. Dieselben sind zeitweise für Wochen oft stark und zwingen den Kranken zur Bettruhe. Dann lassen sie wieder längere Zeit nach, ohne ganz zu verschwinden.

1) Nr. 4. 1898 . 
Allmählich im Verlauf von Jahren stellte sich eine Bewegungsbeschränkung in den Hüftgelenken, der Wirbelsäule und in geringerem Grade auch in den Schulter- und Kniegelenken ein, die immer hochgradiger werdend, zur völligen Versteifung der Hüft- und Wirbelgelenke führten.

Hierdurch sind alle Bewegungen hochgradig behindert. Der Gang ist nur mit Krücken möglich. Auf einem Stuhl kann der Kranke nur sitzen, indem er sich mit dem Kreuzbein am Rande desselben gewissermassen aufhängt. In diesem Stadium scheint die Krankheit abgelaufen zu sein. Die Schmerzen haben aufgehört oder treten wenigstens nur dann auf, wenn durch eine unzweckmässige Bewegung die Knochen der versteiften Gelenke aus ihrer Lage zu einander gebracht werden.

Die Verkrüppelten lernen den Rest der ihnen gebliebenen Bewegung ben utzen und suchen sich damit wiederum im Leben zu bethätigen.

Ein Uebergreifen auf die kleinen Gelenke (Ellenbogen und Handund Fussgelenke) scheint nicht vorzukommen.

Unsere Beobachtung zeigt fast genau dieselben Symptome.

E. P., 30 Jahre alt, kam in meine Beobachtung im Juni 1896 . Er wurde in die medicinische Universitätsklinik im Juli des Jahres 1896 anfgenommen and dort die nachfolgende Krankengeschichte festgestellt.

Vorgeschichte.

Der Vater des Patienten starb in hohem Alter an einem Schlaganfall. Die Mutter und zwei Geschwister leben und sind gesund.

In der Kindheit hat er Masern durchgemacht. Späterhin will derselbe vollkommen gesund gewesen sein und insbesondere nicht an Gelenkerkrankungen gelitten haben.

Im Februar 1890 stürzte er auf einer Treppe, fiel zunächst mit der linken Schlüsselbeingegend gegen einen eisernen Ständer und hierauf mit dem Gesäss auf die Kante der Treppe auf.

Er war nur kurze Zeit, nngefähr 5 Minuten, bewusstlos, konnte sich aber dann allein erbeben.

Zwei bis drei Tage nach dem Unfall entwickelten sich Schmerzen in der Kreuzbeingegend, die sich beim Bücken bemerkbar machten..

Dieselben blieben trotz vielfacher Behandlung die nächsten Jahre unverändert bestehen.

Im Januar 1892 begann dann die rechte Hüfte schmerzhaft zu werden, und beim Gehen fiel dem Patienten eine gewisse Schwerbeweglichkeit derselben auf.

Unter Salicylbehandlung gingen die Beschwerden in den Hüften zurück. Die Schmerzen in der Kreuzbeingegend blieben aber bestehen.

Im Jahre darauf, 1893, stellten sich dieselben Beschwerden in der rechten Hüfte wieder ein und gingen auch durch Behandlung mit Salicylpräparaten nicht mehr zurück. Im Gegentheil, es verstärkten sich die Beschwerden, und die Steifigkeit in der rechten Hüfte ging auch auf den unteren Theil der Wirbelsäule über. 
Spüter warde anch die linke Hüfte befallen.

Die Schmerzen und Steifigkeit in beiden Hüftgelenken and der Wirbelsäule nahmen in den pächsten Jahren immer mehr zu und die Wirbelsäule versteifte allmählich bis zum Kopf hinauf.

Im Jahre 1895 waren die Beschwerden beim Gehen so hochgradig geworden, dass er fast ständig zu Bett liegen musste und sich nur mit grosser Mühe aufrichten und einige Schritte gehen konnte.

Um diese Zeit trat auch eine Einschränkung der Bewegung im rechten Schultergelenk und in beiden Kniegelenken auf, die jedoch nicht erheblich waren.

Im Verlauf des Jahres 1896 liessen die Schmerzen nach und gingen so weit zurück, dass Patient wieder gehen lernte.

Die objectiven Erscheinungen blieben unverändert.

Der Befund, wie er in Juli 1896 in der medicinischen Klinik aufgenommen ist, deckt sich völlig mit dem von mir im Juli 1898 festgestellten.

Die subjectiven Beschwerden sind jetzt aber su weit geschwunden, dass der Kranke nit völlig steifer Wirbelsäule nnd Hüftgelenken gut geht, sich schnell um sich selbst drehen und anch kleine Gegenstiinde rom Erdhoden aufheben kann.

Stat. praes.: Pat., $144 \mathrm{~cm}$ gross, von guter Gesichtsfarbe, mässig genährt. Appetit gut.

Die auffallendste Veränderung bietet beim Patienten die Wirbelsänle dar (Fig. 1).

Dieselbe ist in ihrer ganzen Ausdehnung vollkommen steif und unbeweglich und liisst sich auch gewaltsam nicht im Mindesten biegen.

Sie stellt einen leicht nach vorn gebogenen Stab dar, dessen Biegung in dem Brust- und Halstheile erheblich stärker ist wie in dem fast geraden Lumbaltheil.

Die Wirbelsäule weicht vom Kreuzbein an etwas nach rechts ab und ist in den oberen Theilen entsprechend compensatorisch nach links verbogen.

Auch seitliche Bewegungen sind nicht im



Fig. 1. Allergeringsten ausfïhrbar.

Die Dornfortsätze zeigen keine Anomalie und sind alle gut abtastbar.

In der Höhe des Lendentheils der Wirbelsänle fühlt man $\mathrm{zu}$ beiden Seiten den langen Rückenstrecker bretthart gespannt, von mittlerem Volumen.

Am Kreuzbein nichts Besonderes zn fühlen; das Steissbein ebenfalls fest und normal nach vorne geschwungen. 
Der Kopf ist nach vorne in einem Winkel von $30^{\circ}$ geneigt. Er lässt sich biegen und strecken in einer Breite von $15^{0}$, worauf man auf einen harten, nicht elastischen Widerstand stösst.

Rotationsbewegungen werden etwa in einem Winkel von $10^{\circ}$ (activ und passiv) ausgeführt, dann fühlt man ebenfalls einen nicht elastischen Widerstand. Seitliche Bewegungen sind nicht ausführbar.

Der Thorax ist mässig abgeflacht, die rechte Seite im unteren Theile etwas mehr vorgewölbt, entsprechend der Skoliose.

Die Athmung ist fast rein abdominell, jedoch hebt sich auch ein wenig

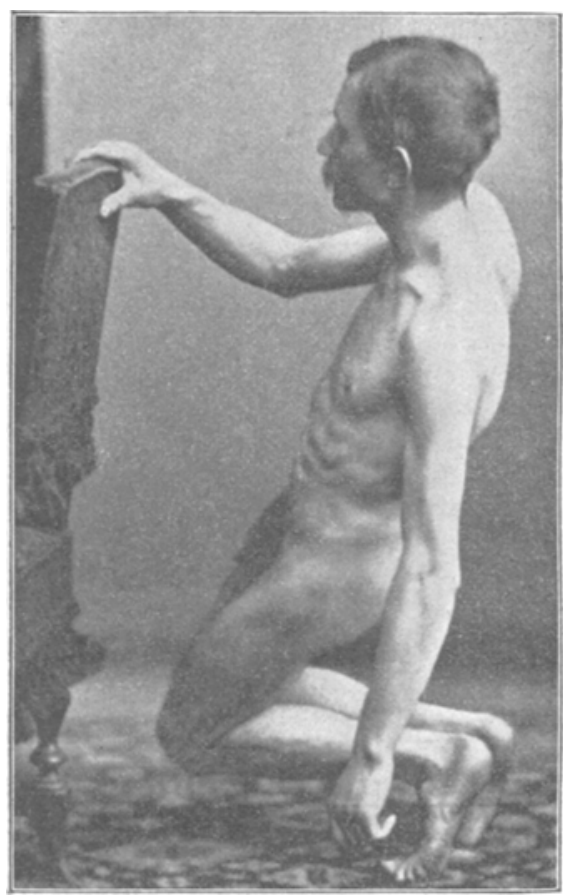

Fig. 2. der Brustkasten. Die Erweiterung bei der Inspiration beträg etwas über $1 \mathrm{~cm}$ (Brustweite $71 \mathrm{~cm}$ ).

Das linke Schlüsselbein ist in der Mitte etwas verdickt (Folge eines alten Bruches). Die Bewegungen am linken Sternoclaviculargelenk sind normal, rechts ist dasselbe etwas verdickt und der Sternalrand des Schlüsselbeins mit einigen Knochenstacheln besetzt.

Die Bewegungen sind etwas beschränkt.

Man fühlt Reiben im Gelenk.

Der linke Arm kann fast bis zur Senkrechten erhoben werden.

Nur bei extremen passiven Bewegungen fühlt man etwas Knarren. Rechts ist die Bewegung im Schultergelenk viel schwerer, die Hebung des Armes ist mit Mühe bis zur Horizontalen möglich, dabei fühlt man Krachen im Gelenk. Ellenbogen- und Fingergelenke sind beiderseits absolut intact.

Eine am linken vierten Finger bestehende Steifigkeit ist auf eine alte Verletzung zurückzuführen. weglich.

Die Hüftgelenke sind in ganz leicht gebeugter Stellung absolut unbe-

Das rechte Bein ist etwas abducirt, das linke entsprechend adducirt, so dass dieselben parallel zu einander stehen.

Dementsprechend ist das rechte Bein etwas länger als das linke und wird das Becken links etwa $1 \frac{1}{2} \mathrm{~cm}$ höher getragen wie rechts.

An den Kniegelenken bemerkt man rechts am inneren Condylns einen knöchernen Auswuchs von der Grösse einer Kirsche. Derselbe hat eine leicht höckerige Oberfläche, setzt sich nach hinten scharf vom Oberschenkel $a b$, während er nach vorne und oben allmählich in ihn übergeht.

Die Bewegung ist in beiden Kniegelenken ein wenig beschränkt und geht nicht viel über $1 \mathrm{R}$ hinaus. 
Beitrag z.chron., ankylosirend.Entzïndg. d. Wirbelsäule u.d.Hüftgelenke. 243

Die Streckung ist normal.

Die Fuss- und Zehengelenke sind vollständig normal.

Der Gang des Patienten ist eigenthümlich.

Er geht nur in den Kniegelenken und streckt den Fuss des jedesmaligen Standbeins extrem im Sprunggelenk.

Er ermı̈glicht hierdurch das Durchschwingen des Gangbeins. Er kommt mit diesem Gange ziemlich schnell vorwärts und vermag mit einem Stocke auch grössere Strecken zurückzulegen.

Ohne Stock äquilibrirt er den unbeweglichen Körper durch Bewegungen mit beiden Armen.

Einen Gegenstand von der Erde aufzuheben, vermag er nur, wenn er einen Haltepunkt mit der Hand findet (Fig. 2).

Er vollführt dann eine Bengung beider Kniee bis auf die Erde und erreicht so mit der freien Hand den Fussboden anch ohne Biegen der Wirbelsäule und Hüftgelenke.

Auf' einen Stuhl kann sich Patient nur mit Mühe setzen. Er beugt dabei die Kniegelenke und lässt sich vorsichtig nach hinten fallen, bis er mit dem Kopf an die Rückenlehne anstösst.

Vor dem Herunterrutschen bewahren ihn die in den Kniegelenken flectirt gehaltenen Unterschenkel.

Auf kurze Zeit kann er sogar anf den Tubera ischii, ohne eine Stütze am Kopfe zu haben, sitzen mit stark unter den Stuhl gezogenen Füssen.

Im Bett liegt er meistens auf den Seiten, kann aber anch mit einem Kopfkissen, das er sich gut unter den Kopf steckt, auf dem Rücken liegen, ohne Schmerzen in der Halswirbelsäule zu bekommen.

Er dreht sich schnell und geschickt um, indem er sich nach soldatischer Art mit einem Fusse abstösst und anf der Hacke des anderen balancirt.

Die Extremitätenmusculatur ist bei dem Patienten gut entwickelt. Händedruck recht kraftvoll.

Sensibilität am ganzen Körper intact. Keine neuralgischen Schmerzen. Bauch-, Cremaster- und Patellarrellexe intact. function.

Kein Fussclonus. Urin, Stuhlgang normal, ebenso die Geschlechts-

Die elektrische Untersuchung der Muskeln zeigt ebenfalls keine $\mathrm{Ab}$ weichung von der Norm.

Die Beobachtungen von Pierre Marie gewinnen nun ein besonderes Interesse durch den Hinweis desselben, dass wir es hier mit einer neuen Krankheit zu thun haben, die bisher unserer Forschung entgangen ist.

Pierre Marie bringt für diese Auffassung allerdings noch ziemlich spärliches Material bei.

DieKrankheiten, zu denen dieSpondylose rhizoméliquegehören könnte, sind der chronische Gelenkrheumatismus und die Arthritis deformans.

Er findet eine Unterscheidung gegen sie in dem Freibleiben der kleinen Gelenke bei seinen Beobachtungen.

Sicherlich ist das ein Punkt ron grosser klinischer Bedeutung nur muss man sich nicht zu starr an diese Definition halten. Denn, 
wenn viele grosse Gelenke von einer Allgemeinkrankheit ergriffen werden, so wird es sicher vorkommen, dass gelegentlich einmal ein kleines Gelenk derselben Veränderung anheimfällt.

Ich kann durch die Güte des Herrn Geh.-Raths Lichtheim einen solchen Fall aus der medicinischen Klinik zu Königsberg mittheilen.

Es sind in demselben die Unterkiefergelenke und wahrscheinlich auch die Zehengelenke von demselben Process ergriffen, wie die Wirbelund Hüftgelenke, und dennoch darf man nach dem Gesammtbilde nicht zweifeln, dass hier ein echter Fall von Spondylose rhizomélique vorliegt.

W. L., 32 Jahre alt, hatte im Alter von 15 Jahren Rheumatismus im rechten Bein und lag 14 Tage daran zu Bett.

Auch später hat er häufig rheumatische Beschwerden gehabt.

Im Jahre 1888 waren die Zehengelenke des linken Fusses (mit Ansnahme der grossen Zehe) stark afficirt und sind seit jener Zeit verunstaltet geblieben.

Vor 6 Jahren bekam Patient Schmerzen im Kreuz und in den Schultern. Sehr bald traten Bewegungsstörungen in beiden Schultergelenken auf, die sich auf der linken Seite einige Wochen früher als auf der rechten ansbildeten.

Einige Monate darauf trat eine Steifigkeit im Kreuz anf, so dass sich Patient nur noch mit Mühe und bald gar nicht mehr bücken konnte.

Die Steifigkeit setzte sich nach oben fort und hat seit 2 Jahren auch die Halswirbelsäule ergriffen, so dass Patient den Kopf gar nicht mehr bewegen kann.

Auch in den Hüftgelenken hat sich seit ungefähr 3 Jahren eine gewisse Steifigkeit herausgebildet, die mässige Gangstörungen verursacht.

Seit 4 bis 5 Jahren ist auch in den Kiefergelenken eine Beschränkung der Kaubewegungen eingetreten, die sich immer mehr ausgebildet hat, so dass Patient seit 2 Jahren die Zahnreihen nur auf eine Entfernung von einigen Millimetern auseinander bewegen kann.

Er kann sich nur kleine Fleisch- und Brodstückchen in den Mund schieben und vermag sie dann mit der Zunge zu zerkleinern.

Status praesens.

Der mittelgrosse Patient befindet sich in einem schlechten Ernährungszustand.

Beim Stehen fällt sofort die absolute Steifheit der Wirbelsäule und die Unbeweglichkeit des Kopfes auf.

Bei Bewegungsversuchen erweist sich die Wirbelsäule als völlig versteift, leicht nach vorne gekrümmt und in ihrem Brust- und Halstheil ein wenig nach links geneigt; der Kopf fast völlig unbeweglich, ganz leichte Bewegungen von vorn nach hinten lassen sich mit demselben ansführen, seitliche und Rotationsbewegungen aber nicht.

Die Wirbel-Rippengelenke sind ebenfalls ankylosirt; bei gewöhnlicher Athmung benutzt Pat. nur das Zwerchfell, nur bei tiefster Einathmung bewegt sich der Brustkasten ein wenig.

In den Schultergelenken ist die active und passive Beweglichkeit gleichmässig in ziemlich hohem Grade beschränkt und schmerzhaft. 
Die Ellbogen-, Hand- und Fingergelenke sind gesund.

In den Hüftgelenken bestehen verhältnissmässig geringfügige $\mathrm{Be}-$ wegungsbeschränkungen.

Die Flexion ist weit über einen rechten Winkel ausführbar und kanm beschränkt, ebenso die Adduction, während die Abduction nur bis zum Winkel von $20^{\circ}$ möglich ist und die Extension nicht bis zur völligen Streckung ausführbar ist.

Die Kniee stehen beiderseits in flectirter Stellung und können nicht gestreckt werden, dabei fehlen beim rechten Bein etwa $20^{\circ}$, beim linken etwa $30^{\circ}$ bis zur völligen Streckung.

Die Flexion ist fast normal. Bei den Bewegungen fühlt man Knirschen; beide Gelenke sind verdickt, die Patella ist fast unbeweglich, die Kapsel ist ungleichmässig verdickt, man fühlt Böckerchen in ihr. Am linken Knie fühlt man dicht unterhalb der Gelenkspalte am Condylus internus tibiae eine kleine kirschkerngrosse Knochenprominenz.

Die Fussgelenke sind beiderseits normal, ebenso auf der rechten Seite die Zehengelenke.

Au linken Fuss ist nur die grosse Zehe intact, die übrigen Zehen sind vollkommen unbeweglich in abnormer Stellung, und zwar befinden sich die ersten Glieder in stark extendirter, die beiden letzten in stark flectirter Stellung.

Die Untersuchung der innern Organe ergiebt an den Lungen normale Verhältnisse, am Herzen ist neben dem 1. Ton überall ein systolisches Geräusch hörbar.

Der Pulmonalton ist nicht accentuirt, die Herzdämpfung nicht vergrössert, der Spitzenstoss liegt noch fingerbreit nach innen von der Mamillarlinie.

Ahdomen ohne Besonderheiten; im Urin kein Eiweiss und kein Zucker.

Die Gehirnnerven bis auf eine Spur Nystagmus normal.

Im Bereich der Spinalnerven fällt eine hochgradige Atrophie der Musculi deltoidei auf.

Die elektrische Reaction ist aber anch bei ihnen normal.

Ein wichtigeres Unterscheidungsmerkmal zwischen der Spondylose rhizomélique einerseits und dem chronischen Gelenkrheumatismus und der Arthritis deformans andererseits scheint mir zu sein, dass bei ersterer als einzig wesentliche Folge der Erkrankung eine Ankylose der Gelenke eintritt. Es muss also in der Natur dieses Processes liegen, dass er jedesmal die Versteifung herbeiführt.

Beim chronischen Gelenkrheumatismus kommt ja auch gelegentlich eine völlige Versteifung eines Gelenkes vor.

Dieselbe ist aber fast nie vollkommen und beruht auf einem $\mathrm{Zu}$ sammenwirken von schmerzhafter Inactivität und starker Schwellung der Gelenkkapsel, und ist ein zufälliger Effect der pathologischen Veränderungen.

Bei der Arthritis deformans ist die Versteifung ebenfalls nux ein zufälliges Ereigniss, bedingt durch Deformationen der Gelenkflächen. 
Von Knochenerkrankungen sind bei der Spondylose rhizomélique nur kleine Auftreibungen in der Nähe der Gelenke ausserhalb des eigentlichen Gelenks wahrzunehmen.

Im klinischen Bilde spielen dieselben eine untergeordnete Rolle.

Die Veränderungen an den Gelenkflächen bei derselben, die sich durch Knirschen und Reiben in Gelenken, die sich im Beginn der Erkrankung befinden, verrathen, können ungezwungen als Folge der beschränkten Bewegungsfähigkeit aufgefasst werden.

Ich betrachte daher folgende Punkte als charakteristisch für die Spondylose rhizomélique und halte dieselben für wichtig genug, um sie als besondere Krankheit betrachten zu müssen.

Die Spondylose rhizomélique führt zu einer Ankylose der betroffenen Gelenke, und dies ist die bei weitem hervorstechendstè Eigenthümlichkeit derselben.

Sie beginnt in der Wirbelsäule, ergreift in zweiter Reihe Hüft-, Schulter- und Knie-Gelenke.

Die kleinen Gelenke des Körpers bleiben fast immer verschont; in den seltenen Fällen, in denen ein kleines Gelenk betroffen ist, führt auch hier der Process zur Ankylose desselben.

Die innern Organe, im Besonderen das Nervensystem, bleiben normal, und letzteres wird auch nicht durch Druck von den erkrankten Stellen der Wirbelsäule aus in Mitleidenschaft gezogen.

Das klinische Bild ist dementsprechend von grosser Einförmigkeit, ein Fall sieht fast genau so aus, wie der andere.

Dies trifft nicht nứ für die bisher erwähnten 5 Fälle zu, sondern kann auch aus der Literatur belegt werden.

Der erste Beobachter dieser Erkrankung, S trümpell 1), theilt 2 Fälle mit, die ganz die gleichen Symptome boten. Dasselbe gilt von den Fällen, die Bäumler ${ }^{2}$ ), Beer ${ }^{3}$ ) und Köhler ${ }^{4}$ ) mittheilen.

Ganz neuerdings bringen Mutterer ${ }^{5}$ ) und Gasne ${ }^{6}$ ) je einen Fall, die dies weiter bestätigen.

Besonders betonen möchte ich noch, dass bei allen Fällen durch die Erkrankung der Wirbelsäule weder das Rückenmark noch die aus demselben austretenden Nerven in Mitleidenschaft gezogen wurden.

1) Bemerkung über die chronische ankylosirende Entzündung der Wirbelsäule und Hüftgelenke. Deutsche Zeitschrift für Nervenheilkunde. XI. Bd. 1897.

2) Ueber chronisch ankylosirende Entzündung der Wirbelsäule. Deutsche Zeitschrift für Nervenheilkunde. Band XII. 2. Heft). und 9 .

3) Ueber Versteifung der Wirbelsäule. Wiener medicin. Blätter. Nr. $\$$

4) Charité-Annalen. 1887.

5) Deutsche Zeitschrift für Nervenheilkunde. Band XIII.

6) La semaine médicale. No. 10. 1899. 
Ich finde hierin einen wesentlichen Unterschied gegen die von Bechterew ${ }^{1}$ ) beschriebenen Fälle von Steifigkeit der Wirbelsäule.

Bei ihnen war neben der Ankylose der Wirbelgelenke heftige Intercostalneuralgie mit Anästhesien im Verlaufe einzelner Intercostalnerven das hervorstechendste klinische Symptom.

Diese Neuralgien waren hervorgerufen durch Druck, der in Folge des krampfhaften Processes auf die Nerven in den Intervertebrallöchern ausgeübt wurde.

Daraus dürfte zu folgern sein, dass bei den Bechterew'schen Fällen die Veränderungen an anderer Stelle auftraten und anderer Natur sind, als bei der Pierre Marie'schen Krankheit.

Diese Annahme findet eine gewichtige Stütze in dem übrigen Symptomenbild der Bechterew'schen Fälle.

Es sind nämlich bei ihnen die übrigen Gelenke, im Besonderen die Hüftgelenke völlig gesund. Ich bin also der Ansicht, dass man auf Grund der klinischen Symptome folgende Krankheiten unterscheiden und als besondere betrachten muss:

1. den chronischen Gelenkrheumatismus,

2. die Arthritis deformans,

3. die Spondylose rhizomélique (Pierre Marie-Strümpell),

4. die Versteifung der Wirbelsäule mit Intercostalnenralgien (Bechterew).

Ueber alie pathologische Anatomie der Spondylose rhizomélique hat Pierre Marie in seiner ersten Arbeit nur eine Vermuthung beigebracht.

In dem Musée Dupuytren hat derselbe die skeletirte Wirbelsänle mit den Rippen und dem Becken eines mïnnlichen Individuums gefunden, die Veränderungen zeigte, wie man sie bei der Spondylose rhizomélique vermuthen darf.

Namentlich das Zusammentreffen von Veränderungen in der Wirbelsäule und in den Hüftgelenken scheint es Pierre Marie wahrscheinlich zu machen, dass dieses skelet von einem Manne herrührt, der an dieser Erankheit gelitten hat.

Die Veränderungen des Skelets in dem Musée Dupuytreu bestehen aus Knochenwucherungen, die vorzüglich an den Lendenwirbelkörpern und an dem Pfannenrande der Hüftgelenke sitzen.

Die Unbeweglichkeit der Wirbelsäule war bedingt durch knöcherne Brücken, die von den Knochenwucherungen aus benachbarte Wirbel verbanden.

An der Brust- und Halswirbelsäule änderte sich das Bild.

1) Bechterew, Deutsche Zeitschrift für Nervenheilkunde. XI. Band. 
Hier sieht man, dass die Bänder der Wirbelsäule (Ligamentum longitudinale anticum, interspinale et apicum) und die Gelenkbänder zwischen den Rippen und der Wirbelsäule verknöchert sind und so die Beweglichkeit der Wirbelsäule und Rippen aufheben.

Wir haben nun gesehen, dass Knochenwucherungen ähnlicher Art bei den Pierre Marie'schen Fällen an der Halswirbelsäule vom Munde aus zu fählen waren.

Auch in unserem Fall bestanden am Oberschenkelknorren und am Schlüsselbein analoge Veränderungen.

Die Verknöcherung der Gelenkbänder ist allerdings beim Lebenden nicht direct zu constatiren.

Es will mir aber scheinen, dass der Befund, der in der Chloroformnarkose an den Hüftgelenken in unserem Falle erhoben worden ist, dafür spricht, dass die Ankylose der Hüftgelenke durch eine Verknöcherung der Bänder bedingt sein könnte.

Dr. Höftman und ich konnten im Jahre 1896 in tiefer Narkose nach Erschlaffung der Musculatur bei unserem Patienten beide Hüftgelenke in fast normalen Grenzen in allen Richtungen ohne erhebliche Gewalt und, ohne dass ein Krachen oder Reiben wahrnehmbar war, bewegen.

Dies schliesst eine Verwachsung des Oberschenkels mit dem Becken aus; ebenso ist es nicht möglich, dass die Unbeweglichkeit durch Knochenwucherungen am Pfannenrande bedingt war, da hierdurch erhebliche Bewegungsstörungen auch in der Narkose zurückbleiben müssten. Hingegen kann man sich vorstellen, dass nicht zu starke Knochenspangen in der Gelenkkapsel jede Beweglichkeit bei erhaltenem Bewusstsein ausschliessen, und dass jeder Versuch einer Bewegung sehr schmerzhaft ist, während in der Narkose diese Verknöcherung der Gelenkkapsel zerbrochen wurde und nun jede Bewegung möglich war. -

Nach der Narkose bestanden grosse Schmerzen in der Hüfte, und als wir am nächsten Tage die Beweglichkeit derselben präfen wollten, hinderte die grosse Schmerzhaftigkeit die Versuche, passive Bewegungen vorzunehmen. Als im Verlauf von 14 Tagen diese Schmerzen verschwanden, bestand die alte Unbeweglichkeit weiter fort.

Ich erwähne ferner, dass eine Resection beider Oberschenkelhälse bei der ersten Beobachtung von Pierre Marie, die einige Jahre, bevor der Kranke in Pierre Marie's Beobachtung kam, von einem Chirurgen ausgeführt war, ebenfalls wieder mit völliger Ankylose der Hüftgelenke geendigt hatte, und dieser ungünstige Ausgang lässt sich auch zwanglos durch Verknöcherung der zurbickgebliebenen Kapsel deuten.

Wir haben dieser Auffassung durch eine Röntgen-Photographie unseres Falles eine weitere Stütze geben können. 
Herr Dr. Ludloff hatte die Güte, dieselbe in der chirurgischen Klinik zu Königsberg anzufertigen.

Die unteren Hals- und oberen Brustwirbel sind ohne Verstärkungsschirm mit einer mittelstarken Röhre aufgenommen. Die Exposition ist in einer Entfernung von $35 \mathrm{~cm} 6$ Minuten lang auf einer RöntgenDoppelplatte erfolgt.

Während man bei normaler Wirbelsäule auf einer Röntgen-Photographie, die ohne Verstärkungsschirm gefertigt ist, die einzelnen Wirbel deutlich von einander abgesetzt sieht, sind in unserem Falle dieselben nicht von einander zu unterscheiden.

Zu beiden Seiten des als hellerer Streifen sichtbaren Wirbelkanals ziehen sich streifenförmige Schatten herunter, die sich seitwärts in die Schatten der Rippenansätze verlieren.

Die Gegend der Tubercula costarum ist auffallend verbreitert.

Die Conturen der Rippen sind in der Nähe der Wirbelsäule nicht scharf, sondern gehen in diffuse Schatten zu beiden Seiten der Wirbelsäule über. Hieraus kann man wohl schliessen, dass die Ligamenta longitudinalia intercostalia et capsularia entweder verkalkt oder verknöchert sind.

Im Februar 1589 berichtet nun Pierre Marie ${ }^{1}$ ) in der Semaine médicale über das anatomische Präparat der Wirbelsäule eines Mannes, der an Spondylose rhizomélique gelitten hatte.

Die Ligamenta longitudinalia des Bandapparats der Gelenkfortsätze der Wirbelkörper und der Rippenköpfehen waren verknöchert. Die Zwischenwirbelscheiben waren intact. Es scheint also zu dem klinischen Bilde auch ein anatomischer Befund zu existiren, der es berechtigt erscheinen lässt, die Apondylose rhizomélique als selbständige Krankheit aufzufassen.

Pierre Marie gebührt das Verdienst, dies znerst besonders hervorgehobea zu haben, während die ersten veröffentlichten Beobachtungen von Strümpell herrühren.

1) La semaine médicale. No. 9. 1899. 